\title{
Epidemiologia de Triatominos en la Provincia de Río Negro
}

\section{Triatomine Epidemiology in the Province of Río Negro}

\section{Epidemiologia de triatomíneos na província de Río Negro}

\author{
Crowley $P^{1,2}$, Labanchi $\mathrm{L}^{1,2}$, Martin Dade ${ }^{2}$, Martin Daniele ${ }^{2}$, Calabro $A^{1}$, Grizmado \\ $C^{1}$, Ochoa $A^{1}$, Paula Hernández ${ }^{1}$, Arezo $M^{1}$, Larrieu $E^{2,3}$ \\ 1 Ministerio de Salud de la Provincia de Río Negro, Laprida 240 CP 8500, Viedma, Argentina. \\ 2 Escuela de Veterinaria y Producción Agroindustrial de la Universidad Nacional de Río Negro, Pacheco \\ 460 CP 8360, Choele Choel, Argentina. \\ 3 Facultad de Ciencias Veterinarias de la Universidad Nacional de La Pampa, Calle 5 y 116 CP 6360, \\ General Pico, Argentina.
}

Correo electrónico: ejlarrieu@hotmail.com

\section{RESUMEN}

Chagas es una enfermedad endemoepidémica de transmisión vectorial en la Provincia de Rio Negro causada por Trypanosoma cruzi, transmitido por insectos vectores denominados triatominos (vinchucas). El programa de control de Chagas de la provincia de Rio Negro certifico, a través de indicadores serológicos y entomológicos, la interrupción de la transmisión vectorial en todo el territorio provincial. Sin embargo, en los últimos años se han generado condiciones que pueden posibilitar la reemergencia de la enfermedad, tal como la eventual domiciliación de vinchucas silvestres, tal el caso de Triatoma patagónica. El objetivo fue evaluar la situación epidemiológica del vector mediante estudios de infestación domiciliaria y peri domiciliaria por triatominos y efectuar análisis de resistencia al principal insecticida que fue aplicado durante muchos años en control. Como resultado se evaluaron 143 viviendas y se colocaron 66 trampas de luz. En el intradomicilio todas las viviendas resultaron negativas, resultando 3 positivas en el peri-domicilio (2.1\%) con capturas de T-infestans y $T$. 
patagonica. Con las trampas de luz no se capturaron triatominos. Los insectos capturados fueron T. infestans y T. patagonica los que resultaron todos negativos a T. cruzi. Los estudios de resistencia a insecticidas mostraron que las colonias siguen siendo susceptibles a deltametrina.

Palabras clave: Chagas, triatoma, epidemiologia, control

\section{ABSTRACT}

Chagas is an endemic epidemic disease of vector transmission in the Province of Rio Negro caused by Trypanosoma cruzi, transmitted by triatomines. The Chagas control program in the province of Rio Negro certified, through serological and entomological indicators, the interruption of vector transmission throughout the province. However, in recent years, conditions have been generated that may allow the re-emergence of the disease, such as the eventual domiciliation of wild vinchucas such as $T$ patagonico. The objective was to evaluate the epidemiological situation of the vector through studies of home and peri-home infestation by triatomines and to carry out resistance analyzes to insecticides that were applied for many years in control. As a result, 143 homes were evaluated and 66 light traps were placed. In the intra-domicile all the dwellings were negative, with 3 being positive in the peri-domicile $(2.1 \%)$ with captures of T-infestans and T. patagonica. No triatomines were captured with the light traps. The captured triatomines were T. infestans and T. patagonica, which were all negative for T. cruzi. Insecticide resistance studies showed that the colonies are still susceptible to deltamethrin.

Key words: Chagas disease, triatoma, epidemiology, control

\section{RESUMO}

Chagas é uma doença epidêmica endêmica de transmissão vetorial na Província de Rio Negro causada pelo Trypanosoma cruzi, transmitido por triatomíneos. O programa de controle de Chagas certificou, por meio de indicadores sorológicos e entomológicos, a interrupção da transmissão vetorial em todo o território provincial. Porém, nos últimos anos, foram geradas condições que podem permitir o ressurgimento da doença, como a eventual domiciliação de vinchucas silvestres como o Triatoma patagonica. 0 objetivo foi avaliar a situação epidemiológica do vetor por meio de estudos de infestação domiciliar e peri-domiciliar por triatomíneos e realizar análises de resistência a 
inseticidas que foram aplicados por muitos anos como controle. Como resultado, 143 casas foram avaliadas e 66 armadilhas luminosas foram colocadas. No intra-domicílio todas as moradias foram negativas, sendo 3 positivas no peri-domicílio (2.1\%) com capturas de T-infestans e T. patagonico. Nenhum triatomíneo foi capturado com as armadilhas luminosas. Os triatomíneos capturados foram T. infestans e T. patagonica, todos negativos para T. cruzi Os estudos de resistência a inseticidas mostraram que as colônias ainda são suscetíveis à deltametrina.

Palavras-chave: doença de Chagas, triatoma, epidemiologia

Fecha de recepción artículo original: 18-07-2021

Fecha de aceptación para su publicación: 29-08-2021

\section{Introducción}

La enfermedad de Chagas, mal de Chagas-Mazza o tripanosomiasis americana, es una enfermedad parasitaria tropical, generalmente crónica, causada por el protozoo flagelado Trypanosoma cruzi $^{(1,2)}$

La Provincia de Río Negro se encuentra ubicada dentro del área endémica para Chagas de la República Argentina. La distribución del vector es irregular, coexistiendo Departamentos libres de triatominos ubicados al oeste de la provincia con otros ubicados al norte y el este como General Roca, Pichi Mahuida, General Conesa, Avellaneda, San Antonio, Valcheta y 9 de Julio, que han presentado históricamente niveles caracterizados como de alta infestación de triatominos.

Las actividades de control fueron iniciadas en 1967 en toda el área endémica (fase de ataque con gamexane como insecticida) extendiéndose esta estrategia hasta 1983 con limitados resultados.

El programa fue restructurado en 1983 iniciándose una nueva fase de ataque, la que estuviera a cargo del área provincial de Salud Ambiental en operativos combinados con el Servicio Nacional de Chagas y municipios de áreas endémicas, con insecticidas piretroides, en los Departamentos Valcheta y 9 de Julio que se extendieron posteriormente a la costa del Río Colorado y, finalmente, a los Departamentos General Roca y El Cuy.

En esta fase se efectuaron, entre 1983 y 2000, 15998 rociados de viviendas y se evaluaron 38025 viviendas detectándose 2774 (7.3\%) con triatominos o rastros de los mismos, evolucionando desde su pico máximo en 1985 (14.8\%) al 0.7\% en el año 2000 (R Bigatti y S Romeo, comunicación personal). 
En 1983, asimismo, se realizaron los primeros catastros serológicos a niños y escolares con fines de diagnóstico precoz y tratamiento.

A partir de 2001, la estrategia central fue la vigilancia domiciliaria mediante búsqueda de insectos o sus rastros a cargo de la red de agentes sanitarios de Atención Primaria de la Salud (APS) complementado por búsqueda mediante la técnica de hora/hombre a cargo de personal de Salud Ambiental del Ministerio de Salud, con rociado selectivo solo a las viviendas positivas, con piretroides (R Bigatti y S Romeo, comunicación personal).

En el último trimestre del año 2001 se logró cumplir con los indicadores establecidos por la Organización Panamericana de la Salud obteniéndose así la certificación internacional de provincia libre de transmisión vectorial, siendo recertificada en 2011 y $2019^{(2,3)}$

Sin embargo, en los últimos años se observaron nuevas situaciones epidemiológicas que pudieran generar condiciones para la reemergencia de la enfermedad, tal como el ingreso de población desde áreas endémicas, la adaptación de triatomas autóctonos silvestres a condiciones de domicilio y dificultades para el control de estas situaciones por resistencia a los insecticidas utilizados.

De tal manera, el objetivo del trabajo fue Identificar la prevalencia de viviendas con T. infestans y T. patagónica en diversos ambientes eco-epidemiológicos de la provincia describiendo su asociación con factores de riesgo tal como migraciones y actividades humanas, evaluando la resistencia que podría haber generado el uso intensivo de insecticidas en la etapa de ataque químico del programa de control ${ }^{(4,5)}$.

\section{Materiales y métodos}

Área de trabajo: se incluyeron actividades en localidades con historial de presencia de T infestans: Choele Choel, Luis Beltran, General Roca y San Antonio Oeste en la Provincia de Rio Negro (Figura 1)

Vigilancia activa a través de la búsqueda domiciliaria de triatominos: se utilizó el método hora/hombre, que consiste en investigar en forma sistemática, prolija y completa, el interior (intradomicilio) y el exterior (peridomicilio) de las viviendas por un hombre durante una hora para determinar: la presencia de Triatominos con posterior identificación de especie.

Captura de triatominos en áreas silvestres y periurbanas: mediante la colocación de trampas de luz en diversos ecotopos (bardas, médanos, vegetación arbustiva, etc.) construidas con cajas de cartón de 30 $\mathrm{cm}$ x $30 \mathrm{~cm}$ y con luz blanca en su interior y cinta adhesiva como pegamento para los insectos. 
Acondicionamiento de insectos: los triatomas capturados se colocaron en recolectores plásticos separados, para su posterior envió a laboratorio:

Laboratorio de Chagas de Salud Ambiental de Choele Choel: examen microscópico directo de las heces de los triatominos con objetivo 10x y 40x para clasificación entomológica y presencia de Trypanosoma cruzi.

Laboratorio de artrópodos y vectores de la Facultad de Ciencias Veterinarias de la UNLP para los estudios de resistencia a insecticidas.

Evaluación de resistencia a insecticidas: se evaluaron los niveles de susceptibilidad/resistencia hacia la deltametrina por parte de los ejemplares de T. infestans y T. patagónica capturados, determinando para ello la dosis necesaria para matar al 50\% de la población en estudio (DL50) según protocolo de la Organización Mundial de la Salud ${ }^{(6)}$. Para ello se utilizaron ninfas de $\mathrm{V}$ estadio de T. infestans y T. patagónica con 12-15 días de ayuno.. El bioensayo en donde se determinará la respuesta binaria (muerto o vivo) con una variable independiente (dosis), consiste en la aplicación tópica de $1 \mu \mathrm{L}$ del principio activo (deltametrina) disuelto en acetona, a distintas dosis, sobre la zona dorsal del abdomen de las ninfas. El ensayo se replicó 3 veces en días distintos bajo las mismas condiciones y con soluciones frescas preparadas durante cada replica.

La DL50 obtenida para las poblaciones de T. infestas y T. patagónica capturadas en terreno fueron comparada con la DL50 obtenida para la colonia de referencia (susceptible a deltametrina). Esta colonia de referencia se constituyó con individuos enviados desde el Centro de Referencia de Vectores (CeReVe), Santa María de Punilla, Córdoba, Argentina ${ }^{(6,7)}$.

Figura 1: Área de distribución de triatominos en la Provincia de Rio Negro

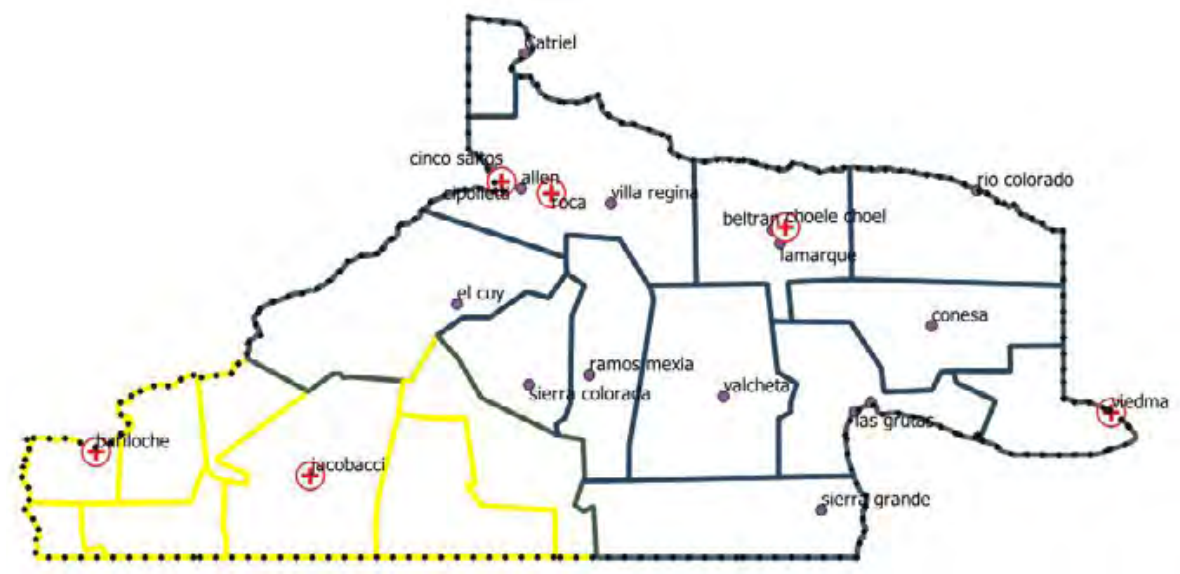




\section{Resultados}

Los resultados de las capturas de insectos tanto por horas nombre como por trampas de luz se presentan en Tabla 1. Un resumen indica que se evaluaron 143 viviendas en las regiones del valle medio (Luis Beltran, Chelforo, Choele Choel y Lamarque), alto valle (General Roca) y costa atlántica (San Antonio Oeste y Las Grutas) y se colocaron 66 trampas de luz en áreas silvestres.

En la evaluación intradomiciliaria todas las viviendas resultaron negativas, resultando 3 positivas en el peri-domicilio (2.1\%). Con las trampas de luz no se capturaron triatominos.

Los triatominos capturados fueron 58 T. infestans y 32 T. patagonica. Asimismo, se recibieron 15 T. patagonica y 5 T. eratyrusiformis, $1 \mathrm{~T}$. platensis traidos por la comunidad.

Todos los triatominos fueron negativos a T. cruzi.

No hubo diferencias entre la dosis letal 50 de la colonia de referencia con las provenientes de Rio Negro. La dosis letal 50\%, expresada en nanogramos x insecto y el grado de resistencia: (DL50 colonia Luis Beltrán / DL50 colonia de referencia) fueron respectivamente 49.2 (IC95\% 34.9-85.4) y 0.66 (IC95\% 0.33-1.3).

Tabla 1: Resultados de búsqueda de triatominos en diversos ambientes en la Provincia de Rio Negro

\begin{tabular}{|c|c|c|c|c|c|c|}
\hline Localidad & \multicolumn{2}{|c|}{$\begin{array}{l}\text { Viviendas } \\
\text { evaluadas* } \\
\mathrm{n}^{\circ}+ \\
\end{array}$} & \multicolumn{2}{|c|}{$\begin{array}{c}\text { Trampas } \\
\text { de luz* } \\
\mathrm{n}^{\circ}+ \\
\end{array}$} & $\begin{array}{l}\text { Triatomas captura- } \\
\text { dos según especie }\end{array}$ & $\begin{array}{l}\text { Triatomas capturados } \\
\text { según localización }\end{array}$ \\
\hline $\begin{array}{l}\text { Choele Choel } \\
\text { y Lamarque }\end{array}$ & 29 & 0 & 20 & 0 & $\begin{array}{l}\text { T. infestans: } \\
\text { T. patagonica: } \\
\text { Otros: } \\
\end{array}$ & $\begin{array}{c}\text { Intradomiciliaria } \\
\text { Peri domiciliaria } \\
\text { Area silvestre } \\
\end{array}$ \\
\hline Luis Beltrán & 36 & $3^{* * *}$ & & & $\begin{array}{cc}\text { T. infestans: } & 58 \\
\text { T. patagonica: } & 32 \\
\text { Otros: } & \\
\end{array}$ & $\begin{array}{c}\text { Intradomiciliaria } \\
\text { Peri domiciliaria: } 90 \\
\text { Area silvestre }\end{array}$ \\
\hline Chelforo & 24 & 0 & & & $\begin{array}{c}\text { T. infestans: } \\
\text { T. patagonica: } \\
\text { Otros: }\end{array}$ & $\begin{array}{c}\text { Intradomiciliaria } \\
\text { Peri domiciliaria } \\
\text { Area silvestre }\end{array}$ \\
\hline $\begin{array}{c}\text { San Antonio } \\
\text { Oeste y Las } \\
\text { Grutas } \\
\end{array}$ & 23 & 0 & 36 & 0 & \begin{tabular}{ll}
\multicolumn{2}{c}{ T. infestans: } \\
T. patagónica: & $7^{* *}$ \\
Otros: & $1^{* *}$
\end{tabular} & $\begin{array}{l}\text { Intradomiciliaria } \\
\text { Peri domiciliaria: } \\
\text { Area silvestre } \quad 7\end{array}$ \\
\hline General Roca & 38 & 0 & 10 & 0 & \begin{tabular}{ll}
\multicolumn{2}{c}{ T. infestans: } \\
T. patagonica: & $7^{* *}$ \\
Otros: & $5^{* *}$ \\
\end{tabular} & $\begin{array}{l}\text { Intradomiciliaria: } \\
\text { Peri domiciliaria: } \\
\text { Area silvestre: }\end{array}$ \\
\hline TOTAL & 1433 & $2.1 \%)$ & 66 & 0 & $\begin{array}{ll}\text { T. infestans: } & 58 \\
\text { T. patagonico: } & 37 \\
\text { Otros: } & 6\end{array}$ & $\begin{array}{c}\text { Intradomiciliaria } \\
\text { Peri domiciliaria: } 90 \\
\text { Area silvestre }\end{array}$ \\
\hline
\end{tabular}

* n ${ }^{\circ}$ número de viviendas evaluadas, + numero de viviendas con presencia de triatominos. ${ }^{* *}$ entregados por pobladores. Otros: 5 T. eratyrusiformis, 1 T. platensis. ${ }^{* * *}$ positivas en peri-domicilio 


\section{Discusión}

En 1914 se descubre en Jujuy, provincia argentina, la existencia de Trypanosomas en un T. infestans, En 1918 se logró la primera comprobación del parasitismo humano en un extendido de sangre tomado de un aborigen de Jujuy.

Si bien existen cerca de 130 especies de triatominos y más de la mitad ha sido encontradas natural o experimentalmente infectadas por T. cruzi, solo una docena tienen importancia epidemiológica para el hombre por su adaptación al ecotopo doméstico y peri doméstico, siendo capaces de colonizar la vivienda y alimentarse de las personas $(1,2)$.

En la Argentina se considera que existen 17 especies de triatominos distribuidas en 3 géneros: Psamolestes, Pastrongylus y Triatoma (especies: breyeri, delpontei, garciabesi, infestans, limai, melanosoma, platensis, eratyrusiformis, rubrovaria, patagónica, guasayana, sórdida) (8)

Estas especies difieren en importancia epidemiológica según su hábitat, densidad poblacional y distribución geográfica. En nuestro país $T$. infestans es una de las especies mejor adaptadas a la vivienda humana, ya que es el único triatomino domiciliado. Esto es debido a su fototropismo, criotropismo e higrotropismo negativos, lo que las hace adaptarse y procrearse favorablemente en la vivienda precaria por lo que reviste mayor importancia epidemiológica. Su distribución geográfica es amplia en Argentina, habiéndose detectado incluso en la Provincia de Chubut, en el sur de la Argentina (9).

Otras como T. guasayana, eratyrusiformis, platensis, patagónica y sórdida, son especies que se encuentran en transición entre el hábitat silvestre y doméstico, utilizando al peri domicilio como primer escalón en su proceso de adaptación al hábitat humano, alimentándose de animales domésticos y peri domésticos pudiendo invadir la vivienda e infectarse con T. cruzi $i^{(8,9)}$.

En la provincia de Rio Negro T. infestans presentaba una importante distribución incluyendo el alto y medio valle del Rio Negro, la costa del Rio Colorado, la costa atlántica con especial referencia a San Antonio Oeste y en un sector del sur provincial, en el límite con la provincia de Chubut, con epicentro en Valcheta y Ramos Mexia. Los porcentajes de triatomas infectados con $T$ cruzi eran elevados y en consecuencia el número de casos crónicos era de importancia sanitaria, aunque sin observase casos agudos de la enfermedad ${ }^{(11)}$.

T. patagonica, por su parte, es el triatoma silvestre adaptado a la región, viviendo en zonas de monte, con ingreso ocasional a viviendas, hasta el momento sin domiciliarse y siendo negativos a T. cruzi. 
Accesoriamente, el programa de control mantiene acciones de vigilancia seroepidemiológica en escolares y grupos de riesgo y vigilancia epidemiológica de la infección en la madre y el niño para la detección de Chagas congénito y el tratamiento oportuno de los casos detectados ${ }^{(12,13)}$

Los resultados de este estudio muestran que programas consistentes con el soporte de sistemas de vigilancia pueden limitar la infestación domiciliaria por T. infestans ${ }^{(14,15)}$.

Resulta de interés constatar que a pesar de largos años de fase de ataque y posterior vigilancia epidemiológica con rociado selectivo con piretroides no ha generado resistencia demostrable a los insecticidas utilizados.

La situación epidemiológica actual es consistente con la certificación del cese de la transmisión vectorial alcanzado en 2001 y sostenido oficialmente en las sucesivas certificaciones, con un muy bajo nivel actual de infestación domiciliaria por triatomas y con ausencia de $T$. cruzi.

Sin embargo, debe continuarse prestando atención a situaciones epidemiológicas y a ecotopos específicos que pueden implicar reemergencia de riesgos como los detectados en el alto valle del Rio Negro en el año 2010 donde estudios efectuados encontraron en zonas de hornos de ladrillos con alta proporción de población migrante de Bolivia que con el encendido de los hornos en horario nocturnos los propietarios encontraban habitualmente triatominos que llegaban volando. En las viviendas de los propietarios, asimismo, se detectaba hasta un $20 \%$ de casas positivas con presencia de rastros, pelechos y/o huevos, ejemplares adultos y ninfas de los estadios I y V de T. infestans lo que implicaba su domiciliación ${ }^{(16) .}$

Asimismo, el presente trabajo permite constatar que T. patagónica, adaptado a los ecotopos de la región y con amplia distribución en Rio Negro, se mantiene como un vector silvestre, no domiciliado y sin impacto en salud. 


\section{Bibliografía}

1. Sosa-Estani S, Segura EL. Integrated control of Chagas disease for its elimination as public health problem--a review. Mem. Inst. Oswaldo Cruz. 2015;110(3):289-98.

2. Salvatella R, Irabedra P, Castellanos LG. Interruption of vector transmission by native vectors and "the art of the possible". Mem. Inst. Oswaldo Cruz. 2014; 109(1):122-5

3. Salerno R, Salvatella R, Issa J, Anzola MC. A regional fight against Chagas disease: lessons learned from a successful collaborative partnership. Rev Panam Salud Publica. 2015; 37(1):38-43.

4. Provecho YM, Fernández MDP, Salvá L, Meli S, Cano F, Sartor P, Carbajal-de-la-Fuente AL. Urban infestation by Triatoma infestans (Hemiptera: Reduviidae), an overlooked phenomena for Chagas disease in Argentina. Mem. Inst. Oswaldo Cruz. 2021; 116:e210056.

5. Gorla DE. Climate change and vector-borne diseases in Argentina. Medicina (B Aires). 2021; 81(3):432-437.

6. WHO (World Health Organization). Protocolo de evaluación de efecto insecticida sobre triatominos. Acta Toxicológica Argentina. 1994; 2:29-32.

7. Dade M, Daniele M, Silvestrini MP, Mestorino N. Eficacia in vitro de tres endectocidas frente a Triatoma infestans.. Rev. Cub. Med.Trop. 2014; 66 (3):338-350.

8. Ceccarelli S, Balsalobre A, Cano ME, Canale D, Lobbia P, et al. Analysis of Chagas disease vectors occurrence data: the Argentinean triatomine species database. Biodivers Data J. 2020; 8:e58076.

9. Piccinali RV, Canale DM, Sandoval AE, Cardinal MV, et al. Triatoma infestans bugs in Southern Patagonia, Argentina. Emerg. Infect. Dis. 2010; 16(5):887-9

10. Lent H, Wygodzinsky PW. Revision of the Triatominae (Hemiptera, Reduviidae), and their significance as vectors of Chagas' disease. Bull. Am. Mus. Nat. His. 1979; 163: 123-520

11. Acevedo J, Mercapide C. Pereyra R, Aguilar S. Megacolon del adulto en la zona atlántica. Rev. Arg. Cirug. 1998; 74:65-72

12. Altcheh J, Castro L, Dib JC, Grossmann U, Huang E, Moscatelli G, et al. Prospective, historically controlled study to evaluate the efficacy and safety of a new paediatric formulation of nifurtimox in children aged 0 to 17 years with Chagas disease one year after treatment. PLoS Negl. Trop. Dis. 2021; 15(1):e0008912.

13. Lascano F, García Bournissen F, Altcheh J. Review of pharmacological options for the treatment of Chagas disease. Br. J. Clin. Pharmacol. 2020;13 Online ahead of print.

14. Cardinal MV, Enriquez GF, Macchiaverna NP, Argibay HD, Fernández MDP, et al. Longterm impact of a ten-year intervention program on human and canine Trypanosoma cruzi infection in the Argentine Chaco. PLoS Negl. Trop. Dis. 2021;12;15(5):e0009389.

15. Abrahan L, Cavallo MJ, Amelotti I. Impact of involving the community in entomological surveillance of Triatoma infestans (Klug, 1834) (Hemiptera, Triatominae) vectorial control. Parasit. Vectors. 2021; 5;14(1):98. 
16. Orellana B. Evaluación de los factores de riesgo para la enfermedad de Chagas en la zona rural de Cinco Saltos y Contralmirante Cordero en la Provincia de Rio Negro. Tesis para la Licenciatura en Saneamiento y Protección Ambiental de la Universidad del Comahue 2010; 72pag 